\title{
Gametophytic-sporophytic incompatibility in the Cruciferae - Brassica campestris
}

\author{
M. I. Zuberi* and \\ D. Lewis
}

School of Biological Sciences, Queen Mary College, Mile End Road, London E1 4NS.

Self-incompatibility in Brassica campestris is controlled by two complementary genes, the long-established $S$ gene with sporophytic action on the pollen and a pollen-gametophytically active gene, $G$, which is complementary to $S$ and is oppositional in its action in that both $S$ and $G$ alleles must be matched to give an incompatible reaction.

The $G$ genes in both Brassica campestris and Raphanus sativus are closely similar. They appear to have, in both species, a low number of alleles, are fully expressed only in some $S$ allele combinations and are linked to $S$. The evidence suggests that $G$ is the forerunner of the gene in the gametophytic system in dicotyledons and has been retained in the sporophytic system as an essential part of the incompatibility process.

\section{INTRODUCTION}

The background to our search for a second gene which complements the well-established sporophytically expressed $S$ gene in the Cruciferae has been given in Lewis et al. (1988). In an earlier paper (Zuberi et al., 1981) the results of one intrafamilial pollination in Brassica campestris could not be explained on the one $S$ gene model. The analysis of further generations derived from this exceptional family is presented here. The results and methods used on the two species, Raphanus sativus and Brassica campestris, are similar and have repeatedly complemented one another. We have, during the work analysed the results with different working hypotheses and have now finalised the analysis after all the data on the two species are available. The second gene, with its gametophytic action which we claim is similar in both species, has given us confidence that we are dealing with an important genetic aspect of the sporophytic incompatibility system.

\section{MATERIAL AND METHODS}

All the plants used in the present study originated from three bud-selfs and six sib-crosses of family

\footnotetext{
* Permanent Address: Department of Botany, University of Rajshahi, Rajshahi, Bangladesh.
}

6/79 which is described in fig. 1, Zuberi et al. (1981). Culture methods were not changed but natural temperatures in 1980 were higher so that the plants were smaller and with fewer flowers than those analysed in 1979. For the grouping of plants in families, the pollination, staining and assessment of the results followed Zuberi et al. (1981). After the classification was completed and a gametophytic gene appeared to fit the results it was apparent that a more quantitative method should be used to check the crosses between assumed genotypes.

A method of restricted pollination was tested by applying pollen to the stigma in the usual way by touching the stigma with a dehisced anther and carefully brushing the stigma to remove all but one layer of pollen grains. Inspection under a binocular microscope, and also counting the number of pollen grains which could be washed off the stigma after brushing, provided an assessment of the testbrushing technique. With standard brushing, the number of pollen-grains left on a stigma varied from 1018 to 356 , mainly because of differences in sizes of stigmas. The important point was that most of the pollen grains were in direct contact with the papillae of the stigma. Eighteen to twenty hours after pollination the stigmas were removed and stirred in a large drop of stain on a slide to remove loose ungerminated pollen. The stigma was left to stain and then squashed. Ungerminated, good and 


\begin{tabular}{|c|c|c|c|c|c|}
\hline Pollen & & $S_{4,4}{ }^{(1)}$ & \multicolumn{3}{|c|}{$S_{3, \dot{4}}(10)$} \\
\hline Stigma & $G_{\text {homo }}^{1,1} G_{1,2} G_{\text {homo }}^{2,2,}$ & $G_{1,1}$ & $G_{2,2}$ & $G_{1,2}$ & $G_{1,2}$ \\
\hline$S_{3,3} \begin{array}{l}G_{1,1} \\
G_{1,2} \\
G_{1,1}\end{array}$ & - & + & + & + & + \\
\hline$S_{4,4} G_{1,1}$ & + & - & +- & +- & +- \\
\hline$S_{3,4} \begin{array}{l}G_{2,2} \\
G_{1,2} \\
G_{1,2}\end{array}$ & - & - & $\begin{array}{l}- \\
- \\
-\end{array}$ & $\begin{array}{l}+- \\
- \\
-\end{array}$ & $\begin{array}{l}+- \\
- \\
-\end{array}$ \\
\hline
\end{tabular}

Figure 1 Mating matrix of Fam. 6/79 simplified from fig. 2, Z,Z\&L, 1981. The family was derived from bud-selfing and contained 5 mating groups. It is here represented as 3 major $S$ groups and three minor sub-groups which are allocated genotypes of gametophytic gene $G$.

- , all incompatible; + , all compatible; +- , semi-compatible. $12=$ Number of plants. Plant 13 and 19 are in the $S_{3,4}$, $G_{2,2}$ class.

any small aborted pollen grains were counted in the washing stain, and on the squashed stigma for compatible $(+)$ pollen grains (empty and with penetrating tubes) and incompatible $(-)$ (full and darkly stained and some with short unpenetrating tubes). Counts of aborted and good pollen were also made directly from dehisced anthers. After some experience with the technique it was realised that the counts of free pollen in the washing stain were of little significance and were not made on many of the later tests. Even the percentage of aborted grains could be estimated from the stigmas because they appeared to adhere to the stigma as well as good ungerminating pollen. To keep the counts and assessments as objective as possible all the pollinations were done by M. I. Zuberi, given a code number unknown to $D$. Lewis, who made the preparation and scored all the material for one day without knowing the details of pollination.

Pollen counts from mass but not restricted pollination have been made in Brassica and Raphanus by Tatebe (1940) and Sampson (1962) to show the number of pollen grains on the stigma after self and cross $S$ compatible pollinations. Cornish et al. (1979) used an aerial dust to great effect to obtain accurate counts in the grass Lolium perenne and were able to distinguish four different proportions of + and - pollen. This is the nearest comparison to the restricted brush method.

\section{THE EXCEPTIONAL FAMILY 6/79}

The detailed description of the diallel pollination results between the twenty-three plants in the exceptional family 6/79 derived by bud-selfing $S_{3,4}$ was given in Zuberi et al. (1980). Three major mating groups were found as expected, and one of the major groups was subdivided into two subgroups, and there were two exceptional plants, 13 and 19 . The possibility of pollen contamination during bud-selfing was considered in detail but failed to explain these exceptional plants. The results which we now describe show that all the plants of the family are either $S_{3,3}, S_{4,4}$ or $S_{3,4}$ and no other $S$ allele is present. The exceptional results cannot be fitted to a second gene with sporophytic control but do fit satisfactorily to a second gene $G$, complementary to $S$, and with gametophytic action as outlined for Raphanus sativus in Lewis et al. (1988). For ease of presentation we have placed the $G$ genotypes on the simplified matrix given in fig. 1 before the evidence is presented.

\section{RESULTS}

\section{Mating tests on families derived from Fam. 6/79}

The results of three bud-selfed and six crossed families are given in summary form below. They are grouped into three groups.

(1) bud-selfed in which neither $S$ nor $G$ genes are operating and therefore presenting no restriction to the pollen genotypes, allowing free transmission to the next generations.

(2) Crosses which are $S$ compatible and in which the $G$ gene is silent because an incompatible recognition depends upon both $S$ and $G$ alleles being matched.

(3) Crosses in which the $S$ combination is matched and the final recognition depends. upon whether $G$ is matched or not.

\section{Bud-pollination-S and $G$ not operating}

Fam. 1/80. Plant $6^{13} / 79 S_{3,4} \quad G_{2,2}$ bud-selfed. This is one of the exceptional plants of Fam. 6/79. The ten plants when intersib pollinated revealed three major groups (fig. 2). When tested against the $S$ genotypes of families, $3 / 80$ (reciprocally) and $2 / 80$ (as males only) these groups proved to be $S_{3,3}, S_{4,4}$ and $S_{3,4}$. No matings of the +- type which are characteristic of $G_{\text {homo }} \times G_{1,2}$ matings were found as expected from the presumed $G$ homozygosity of the parent. There are, however, six compatible matings 


\begin{tabular}{|c|c|c|c|c|c|c|c|c|c|c|c|}
\hline \multirow{2}{*}{\multicolumn{2}{|c|}{ Stigma }} & \multicolumn{2}{|c|}{$S_{3,3}$} & \multirow{2}{*}{$\begin{array}{c}S_{4,4} \\
7\end{array}$} & \multicolumn{2}{|c|}{$S_{\underline{3}, 4}$} & \multicolumn{5}{|c|}{$S_{3,4}$} \\
\hline & & 2 & 9 & & 4 & 5 & 8 & 10 & 1 & 3 & 6 \\
\hline & 2 & & & & - & - & + & + & + & + & + \\
\hline$S_{3,3}$ & 9 & - & - & & - & - & + & + & + & + & + \\
\hline$S_{4,4}$ & 7 & + & + & $\frac{\mathscr{e}}{\vec{E}}$ & - & - & - & - & - & - & - \\
\hline$S_{3,4}$ & 4 & & - & $\frac{\varrho}{\tilde{E}}$ & & - & - & & + & + & + \\
\hline & 8 & - & - & & - & - & - & - & - & - & - \\
\hline$S_{-}$ & 10 & $\begin{array}{l}+ \\
-\end{array}$ & - & & - & - & - & - & $\begin{array}{l}- \\
-\end{array}$ & - & - \\
\hline & 3 & - & - & & - & - & - & - & - & - & - \\
\hline & 6 & - & - & & - & - & - & - & - & - & - \\
\hline
\end{tabular}

Figure 2 Fam. 1/80, selfed in bud $6^{13} / 79\left(S_{3,4} G_{2,2}\right)$. Note change of $S_{3,4}$ dominance and absence of +- . The two matings between plants 4 and 5 as females with plant 8 as male cannot be explained without introducing some new factor.

\begin{tabular}{|c|c|c|c|c|c|c|c|c|c|}
\hline \multirow{2}{*}{$\begin{array}{l}\text { Pollen } \\
\text { Stigma }\end{array}$} & $S_{3,3}$ & \multirow{2}{*}{\multicolumn{2}{|c|}{$\begin{array}{l}S_{4,4} \\
G ?\end{array}$}} & & \multicolumn{5}{|c|}{$S_{3,4}$} \\
\hline & $G ?$ & & & & \multicolumn{3}{|c|}{$G$ homo } & \multicolumn{2}{|c|}{$G_{1,2}$} \\
\hline$S_{3,3}$ & $\begin{array}{l}-- \\
-- \\
--\end{array}$ & $\begin{array}{l}+\quad+ \\
+\quad+ \\
+\quad+\end{array}$ & & $\begin{array}{l}+ \\
+ \\
+\end{array}$ & $\begin{array}{l}++ \\
+\quad+ \\
+\end{array}$ & $\begin{array}{l}+ \\
+ \\
+\end{array}$ & $\begin{array}{l}+ \\
+ \\
+\end{array}$ & $\begin{array}{l}+ \\
+ \\
+\end{array}$ & $\begin{array}{l}+ \\
+ \\
+\end{array}$ \\
\hline$S_{4,4}$ & $\begin{array}{l}++ \\
++\frac{0}{5} \\
++ \\
++ \\
+\end{array}$ & $\begin{array}{ll}- & - \\
- & - \\
- & - \\
- & -\end{array}$ & - & $\begin{array}{l}- \\
- \\
-\end{array}$ & $\begin{array}{ll}- & - \\
- & - \\
- & -\end{array}$ & $\begin{array}{l}- \\
- \\
-\end{array}$ & $\begin{array}{l}- \\
- \\
- \\
-\end{array}$ & $\begin{array}{l}- \\
-\end{array}$ & $\begin{array}{l}- \\
- \\
-\end{array}$ \\
\hline$S_{3,4}$ & $\begin{array}{c}-- \\
-- \\
-- \\
-- \\
- \\
- \\
-\end{array}$ & $\begin{array}{l}- \\
- \\
+- \\
- \\
- \\
- \\
+-\end{array}$ & & $\begin{array}{l}- \\
- \\
- \\
- \\
- \\
-\end{array}$ & $\begin{array}{ll}- & - \\
- & - \\
- & - \\
- & - \\
- & -\end{array}$ & $\begin{array}{l}- \\
- \\
-\end{array}$ & $\begin{array}{l}+ \\
+ \\
+ \\
-\end{array}$ & $\begin{array}{l}-+ \\
-+ \\
-+ \\
- \\
- \\
-\end{array}$ & $\begin{array}{l}+- \\
+- \\
+- \\
- \\
- \\
-\end{array}$ \\
\hline
\end{tabular}

Figure 3 Mating matrix of family $2 / 80$ raised from bud-selfing plant $6^{3} / 79\left(S_{3,4} G_{1,2}\right)$. Note the +- results in the $S_{3,4} \times S_{3,4}$ square. G? - constitution unknown, within the $S_{\dot{3}, \dot{4}} \times S_{3, \dot{4}}$ combination. Plants 4 and 5 which are compatible as females with $S_{3,4}$ but not with plant 8 are incompatible as males onto $S_{3,3}$. This can be completely explained if we assume that the two plants have changed $S$ dominance from $S_{3,4}$ to $S_{\underline{3}, 4} ; S_{3}$ has become codominant in the pollen and dominant in the stigma. There remains one anomalous compatible reaction between plant $10, S_{3,4}$ and plant $2, S_{3,3}$. This we cannot explain. The results of this family prove that the exceptional plant 13 is $S_{3,4}$ and not an $S_{\mathrm{x}}$ contaminant, and are consistent with the assumption that it is also homozygous for $G$.

Fam. 2/80. Plant $6^{3} / 79 \quad S_{3,4} \quad G_{1,2}$ bud-selfed. Three major groups are revealed (fig. 3) which are proved to be $S_{3,3}, S_{4,4}$ and $S_{3, \underline{4}}$ by crosses onto $S_{3,3}$ family $3 / 80$ and $S_{3,3} S_{4,4}$ and $S_{3,4}$ of families $6 / 80$ and $7 / 80$. There are nine +combinations within the $S_{3, \underline{4}} \times S_{3, \underline{4}}$ pollinations which split the $S_{3, \dot{4}}$ group into two, corresponding to $G_{1,2}$ and $G$ homozygotes. There are also two +- pollinations in the $S_{3,4} \times$
$S_{4,4}$ combination. Both fully support the $G_{1,2}$ genotype assumed of the parent.

Fam. 3/80. Plant $6^{11} / 79 S_{3,3} G_{1,2}$ bud-self. This is an important test family because it can confirm or otherwise not only the $G_{1,2}$ genotype of plant 11 and the $G$ homozygosity of plant 13 but it will also show the degree of expression of $G$ in an $S_{3,3} \times S_{3,3}$ combination. Eight weak plants were raised, two failed to flower and the remaining six formed one cross incompatible group. Thirty-four of the pollinations were - incompatible and two were repeatedly +- . These involved two plants as female and two others as male. We were able therefore to show $G$ segregation; the two plants used as male were $G_{1,2}$ and the two other plants as female were $G$ homozygous. Test crosses with families $6 / 80$ and $7 / 80$, to be described later, froved that the plants of this family were all $S_{3,3}$ and either $G_{1,2}$ or $G$ homozygous. This family shows that although $G$ expression is rare in the $S_{3,3} \times S_{3,3}$ combination, it does occur. 
Cross pollination-S not matched (compatible), G silent

Fam. 4/80. Plant $6^{8} / 79 \quad S_{3,3} G_{2,2} \times 6^{15} / 79 \quad S_{3,4}$ $G_{1,2}$. In the complete pollination between eleven plants, two major groups, $S_{3,3}$ (6 plants) and $S_{3,4}(5$ plants), were found and crosschecked with plants in other families. Three of the $S_{3,4}$ plants had a dominance change to $S_{3,4}$. Seven +- results were found within the $S_{3,3} \times S_{3,4}$ combination confirming the $G$ genotypes of the parents and the unimpeded transmission of both $G_{1}$ and $G_{2}$ through pollen as expected.

Fam. 5/80. Plant $6^{12} / 79 S_{3,3} G_{1,2} \times 6^{13} / 79 S_{3,4}$ $G_{2,2}$. In the complete pollination between ten plants two major groups were found, $5 S_{3,3}$ and $5 S_{3,4}$. There were six +- results in the $S_{3,3} \times S_{3,3}$ combination (fig. 4). The pattern of results is explained if all the $S_{3,4}$ and three of the $S_{3,3}$ plants are $G_{1,2}$, and two $S_{3,3}$ plants are $G_{2,2}$. Apart from the disparity of the numbers of $G_{1,2}$ and $G_{2,2}$ this agrees with expectations and again confirms the genotype of the exceptional plant $6^{13} / 79$.

Fam. 8/80. Plant $6^{16} / 79 \quad S_{3,3} G_{1,2} \times 6^{19} / 79 S_{3,4}$ $G_{2,2}$. The pollination matrix of nineteen plants was not completed but was sufficient to show that two groups $S_{3,3}$ and $S_{3,4}$ were present and + - results showed that $G_{1,2}$ and $G_{2,2}$

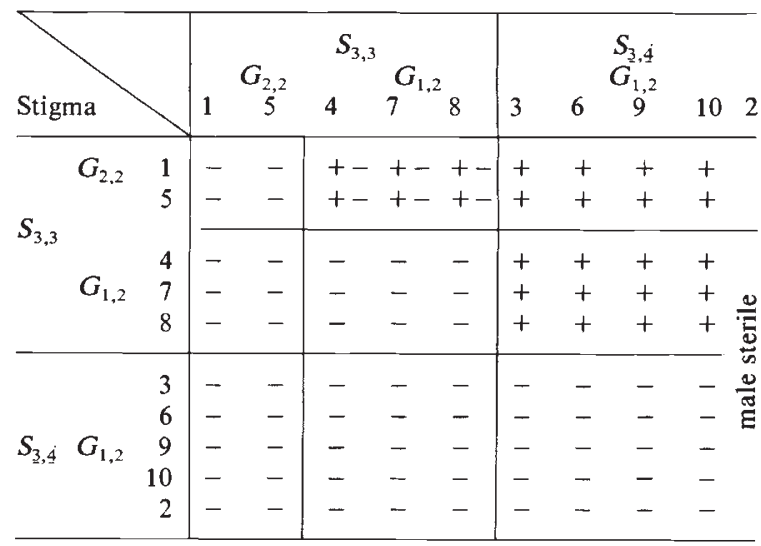

Figure 4 Fam. 5/80, $6^{12} / 79\left(S_{3,3}, G_{1,2}\right) \times 6^{13} / 79\left(S_{3,4} G_{2,2}\right)$. Note +- matings in $S_{3,3} G_{2,2} \times S_{3,3} G_{1,2}$ square. were segregating. This confirms the genotype of the second exceptional plant of family $6 / 79$.

Fam. 9/80. Plant $6^{10} / 79 \quad S_{4,4} \quad G_{1,1} \times 6^{8} / 79 \quad S_{3,3}$ $G_{2,2}$. The pollination matrix of thirteen plants was completed and revealed one cross-incompatible group with no exceptions and no + combinations. Crossing to known genotypes of families $3 / 80,2 / 80$, $6 / 80$, and $7 / 80$ confirmed that all plants were $S_{3,4}$. The lack of +results is expected because all plants should be $G_{1,2}$. The $G_{1,1}$ genotype of $6^{10} / 79$ had been inferred from the +- result obtained with $S_{3,4} G_{1,2}$ plants in the original $6 / 79$ matrix (fig. 1). The $G_{2,2}$ genotype of $6^{8} / 79$ had been assumed from the results of the original 6/79 matrix and from the results of family $6 / 80$.

\section{Cross pollination-S matched and incompatible, $G$ active and compatible}

Fam. 6/80. Plant $6^{13} / 79 \quad S_{3,4} \quad G_{2,2} \times 6^{4} / 79 \quad S_{3,4}$ $G_{1,2}$. The incomplete pollination matrix is given in fig. 5. The major groups $S_{3,3}, S_{4,4}$ and $S_{3,4}$ are present with a deficiency of $S_{4,4}$. There are no +- results in the - blocks, as expected.

Fam. 7/80. $\quad 6^{19} / 79 S_{3,4} G_{2,2} \times 6^{20} / 79 S_{3,4} G_{1,2}$. The complete matrix of twelve plants gave 4 plants of $S_{3,3}, 2$ of $S_{4,4}$ and 6 of $S_{3,4}$. There were no +- results and a good fit to the three $S$ groups with a hint of $S_{4,4}$ deficiency.

These two families not only confirm the genotypes of the two exceptional plants 13 and 19 but give strong support to the gametophytic action of $G$. With $G_{1,2}$ in the pollen and the $S$ gene matched $G_{1}$ only would function on a $G_{2,2}$ stigma, thus all the progeny would be $G_{1,2}$ and no +results.

\section{Intra-familial tests}

Many isolated cross-pollinations between selected plants of different families were made throughout the work in order to keep a cross-check on $S$ and $G$ genotypes allocated to individual plants and incompatibility groups. But four important families were completely cross-checked, using the method of restricted pollination followed by 


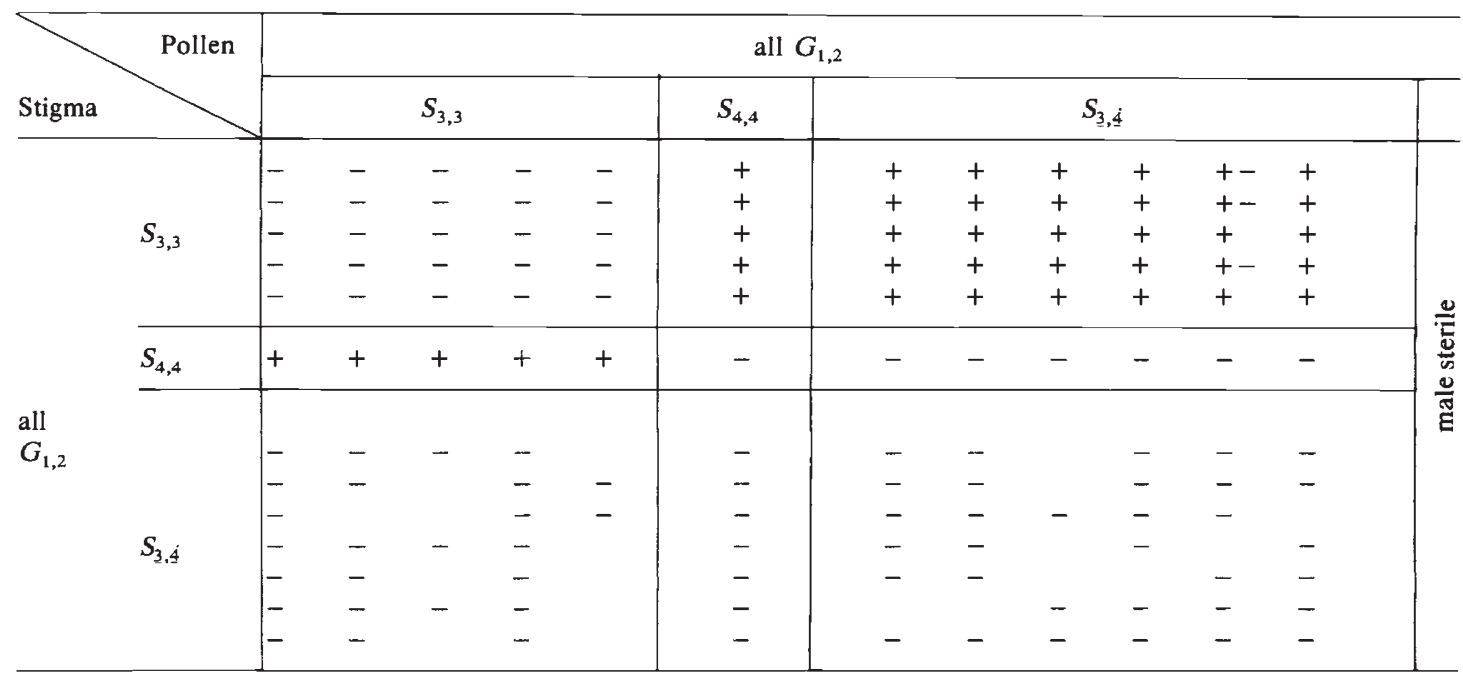

Figure 5 Fam. $6 / 806^{13} / 79\left(S_{3,4} G_{2,2}\right) \times 6^{4} / 79\left(S_{3,4} G_{1,2}\right)$. Note: all plants are $G_{1,2}$ and there are no +- matings in squares.

counting the compatible and incompatible pollen on the stigma, fully described in the next section. The families used on the stigmatic side were fam. $3 / 80\left(S_{3,3} G_{1,2}\right)$ bud-selfed, and all the $S_{3,4}$ progeny of fam. $2 / 80$. On the pollen side, $S_{3,3} G_{1,2}$ plants and $S_{3,4} G_{1,2}$ plants of fams $6 / 80$ and $7 / 80$ were used. The results, given in fig. 6 clearly show the expression of $G_{1,2}$ and $G$ homozygotes segregating. The results are quite clear in the $G_{1,2} \times G_{1,2}$ crosses; without exception they are - incompatible. Most of the $G$ homozygotes $\times G_{1,2}$ crosses are +- as expected but there are some incompatible results which can only be explained by the action of a modifier gene. The action of such a modifier is also required to explain the fact that only two +crosses were found within the family $3 / 80$ pollinations whereas thirteen are found in the intra-family tests.

\begin{tabular}{|c|c|c|c|c|c|c|c|c|c|c|c|c|c|}
\hline \multirow[b]{2}{*}{ Stigma } & \multirow{2}{*}{\multicolumn{2}{|c|}{ Pollen }} & \multicolumn{5}{|c|}{$6 / 80 S_{3,3} G_{1,2}$} & \multicolumn{4}{|c|}{$7 / 80 S_{3,3} G_{1,2}$} & & \\
\hline & & & 2 & 5 & 6 & 9 & 12 & 11 & 2 & 5 & 13 & & \\
\hline \multirow{6}{*}{$3 / 80$} & \multirow{3}{*}{$\begin{array}{l}S_{3,3} \\
G_{1,2}\end{array}$} & 5 & & - & - & - & - & - & - & - & - & & \\
\hline & & 6 & - & - & - & - & - & - & - & - & - & & \\
\hline & & 7 & - & - & - & - & - & - & - & - & - & & \\
\hline & \multirow{3}{*}{$\begin{array}{l}S_{3,3} \\
G_{\text {homo }}\end{array}$} & 3 & $1: 1$ & & & - & - & & & - & - & & \\
\hline & & 4 & $1: 1$ & - & - & - & - & $1: 1$ & $1: 1$ & - & $1: 1$ & & \\
\hline & & 8 & $1: 1$ & $1: 1$ & $1: 1$ & $1: 1$ & $1: 1$ & $1: 1$ & - & $1: 1$ & $1: 1$ & & \\
\hline & \multirow{2}{*}{\multicolumn{2}{|c|}{ Pollen }} & \multicolumn{6}{|c|}{$6 / 80 S_{3,4} G_{1,2}$} & \multicolumn{5}{|c|}{$7 / 80 S_{3,4} G_{1,2}$} \\
\hline Stigma & & & 1 & 3 & 4 & 7 & 10 & 13 & 3 & 4 & 6 & 8 & 10 \\
\hline \multirow{7}{*}{$2 / 80$} & & 3 & - & - & - & - & - & - & - & - & - & - & - \\
\hline & $S_{3, i}$ & 6 & - & - & - & - & - & - & - & - & - & - & - \\
\hline & $G_{1,2}^{3,4}$ & 9 & - & - & - & - & - & - & - & - & - & - & - \\
\hline & & 14 & - & - & - & - & - & - & - & - & - & - & - \\
\hline & & 7 & $1: 1$ & $1: 1$ & $1: 1$ & $1: 1$ & - & $1: 1$ & $1: 1$ & $1: 1$ & - & $1: 1$ & $1: 1$ \\
\hline & $S_{3,4}$ & 11 & - & - & $1: 1$ & $1: 1$ & - & - & - & $1: 1$ & - & $1: 1$ & - \\
\hline & $\vec{G}_{\text {homo }}^{-4}$ & 13 & $1: 1$ & $1: 1$ & $1: 1$ & $1: 1$ & $1: 1$ & $1: 1$ & $1: 1$ & + & + & $1: 1$ & $1: 1$ \\
\hline
\end{tabular}

Figure 6 Fams $3 / 80$ and $2 / 80$ tested with proved $S$ and $G$ genotypes of fams $6 / 80$ and $7 / 80.1: 1=$ approximately equal numbers of + and - pollen grains. 


\section{Pollen counts from restricted pollination}

All the pollinations for the test described in the previous sections were made by a liberal application of pollen to the stigma with several thousand pollen grains, the majority of which would not be in direct contact with the stigma and therefore did not germinate. After we adopted the gametophytically operating $G$ gene to explain the exceptional results both in Brassica and Raphanus, we used the restricted pollination technique described in materials and methods in which only hundreds instead of thousands of pollen grains are left on the stigma. This was essential in order to be able to distinguish late germinated compatible pollen from incompatible pollen with its short nonpenetrating tube.

Table 1 shows the accuracy and some limitations of the technique. All the crosses listed here had been classified as fully incompatible by tests described previously and were expected to be so on the $S$ genotype and lack of $G$ segregation. All but three doubtful pollen grains were incompatible with the characteristic short tube. The three doubtful pollen grains were possibly the result of a damaged papilla which allows penetration of incompatible tubes. The number of pollen grains per stigma varied from 245 to 1568 . Much of this variation was due to the size of the stigma; plant $2^{5}$ had the largest stigma and the highest number of pollen grains, plant $3^{4}$ had the smallest with the lowest number. There is also variation in the per-

Table 1 Numbers of pollen grains found on stigmas and washing fluid after restricted pollination in seven different incompatible crosses. E+ = empty pollen grains with penetrating compatible pollen tubes. $\mathrm{F}-=$ full pollen grains with no or non-penetrating tubes

\begin{tabular}{lrrrrrr}
\hline & \multicolumn{2}{c}{ Stigma } & & & \\
\cline { 2 - 4 } Incompatible cross & E + & F- & \multirow{2}{*}{$\begin{array}{l}\text { Washing } \\
\text { fluid }\end{array}$} & Total $\%$ Germ \\
\hline $2^{5}\left(S_{4,4}\right) \times 6^{4}\left(S_{3,4}\right)$ & 0 & 193 & 825 & 1018 & $18 \cdot 9$ \\
$2^{5}\left(S_{4,4}\right) \times 6^{4}\left(S_{3,4}\right)$ & 0 & 534 & 734 & 1568 & $34 \cdot 0$ \\
$2^{14}\left(S_{3,4}\right) \times 7^{4}\left(S_{3,4}\right)$ & 0 & 244 & 112 & 356 & $72 \cdot 6$ \\
$3^{7}\left(S_{3,3}\right) \times 6^{2}\left(S_{3,3}\right)$ & 0 & 458 & 44 & 502 & $91 \cdot 2$ \\
$3^{7}\left(S_{3,3}\right) \times 6^{2}\left(S_{3,3}\right)$ & 0 & 568 & 197 & 765 & $74 \cdot 2$ \\
$3^{6}\left(S_{3,3}\right) \times 6^{2}\left(S_{3,3}\right)$ & 0 & 761 & 137 & 878 & $84 \cdot 7$ \\
$3^{6}\left(S_{3,3}\right) \times 7^{1}\left(S_{3,3}\right)$ & $? 1$ & 500 & 342 & 843 & $59 \cdot 3$ \\
$3^{6}\left(S_{3,3}\right) \times 7^{1}\left(S_{3,3}\right)$ & 0 & 387 & 170 & 557 & $69 \cdot 4$ \\
$3^{6}\left(S_{3,3}\right) \times 7^{1}\left(S_{3,3}\right)$ & 0 & 333 & 94 & 427 & $77 \cdot 9$ \\
$3^{6}\left(S_{3,3}\right) \times 7^{1}\left(S_{3,3}\right)$ & 0 & 133 & 564 & 697 & $19 \cdot 0$ \\
$3^{4}\left(S_{3,3}\right) \times 7^{2}\left(S_{3,3}\right)$ & 0 & 124 & 188 & 312 & $39 \cdot 7$ \\
$3^{4}\left(S_{3,3}\right) \times 7^{13}\left(S_{3,3}\right)$ & $? 2$ & 67 & 176 & 245 & $27 \cdot 3$ \\
Total & & 4302 & & 8168 & $52 \cdot 6$ \\
\hline
\end{tabular}

* Plant 5 of family $2 / 80$ etc. centage of germination but this does not affect the validity and meaning of the results; the mean germination, 52.7 per cent is similar to that of self-pollination and less than that of compatible cross-pollinations. This difference is significant in the assessment of some critical crosses.

\section{Pollen abortion}

All the families contained some plants which on inspection appeared to have approximately 50 per cent of small empty aborted pollen grains. Aborted pollen grains were counted both directly from the anther and from pollinated stigmas in plants of families $6 / 80,7 / 80,2 / 80$ and $3 / 80$, which were families to be used for restricted pollination. The plants could be readily classified into two groups, (1) plants with less than 5 per cent aborted pollen, and (2) plants which had aborted pollen ranging from 50 per cent to 36 per cent. No plants were found with more that 50 per cent aborted pollen. Because we have found no correlation between the percentage of aborted pollen and percentage of compatible pollen on a stigma in this class, we have presumed the variation has a trivial cause and have called this class 50 per cent aborted pollen (see fig 7(d)).

\section{Cross pollinations-S compatible $G$ silent}

Matings which are compatible in respect of the sporophytically operating $S$ gene, e.g., $S_{3,3} \times S_{3,4}$ and $S_{3,3} \times S_{4,4}$, should ideally give 100 per cent compatible pollen grains which after 24 hours appear as empty pollen grains with penetrating tubes, designated E+. There should be no full pollen grains with non-penetrating tubes designated $\mathrm{F}-$. There may be compatible pollen grains which, due to late germination, have penetrating tubes and yet have considerable cytoplasm and the nucleus still in the pollen grain; these are designated $F$. The results given in table 2 show that the percentage of the three classes of pollen varies from $61 \cdot 4-92 \cdot 2$ per cent $\mathrm{E}+, 7 \cdot 7-37 \cdot 7 \mathrm{~F}$, and nil $\mathrm{F}-$ (see fig. 7(b)). The percentage of germination varies from $95 \cdot 2-65 \cdot 7$. These data are in full agreement with expectations and with the data on Japanese Radish by Tatabe (1940) who found, with compatible crosses, 47-64 per cent of E+, 36-53 per cent of $\mathrm{F}$ and no $\mathrm{F}-$. The percentage germination was 95 per cent. The significantly lower figure for $\mathrm{E}+$ is probably due to the fact that Tatabe did not use restricted pollination; this would cause considerable crowding and competition which would delay germination. 
(a)

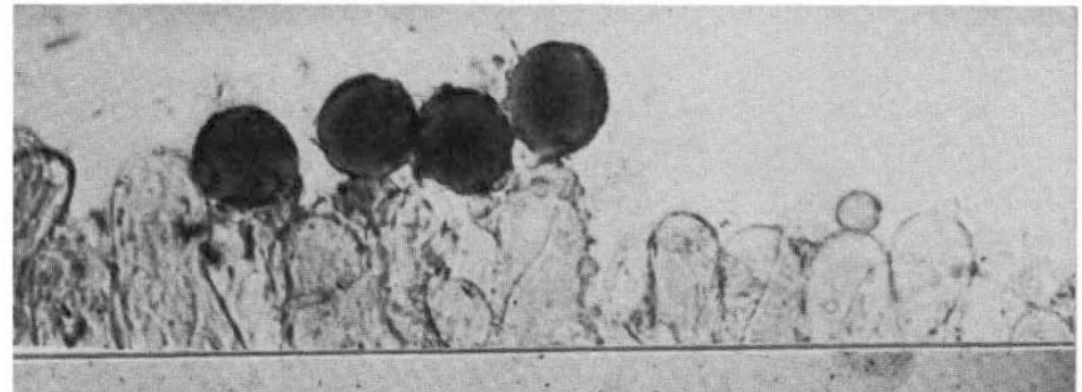

(b)

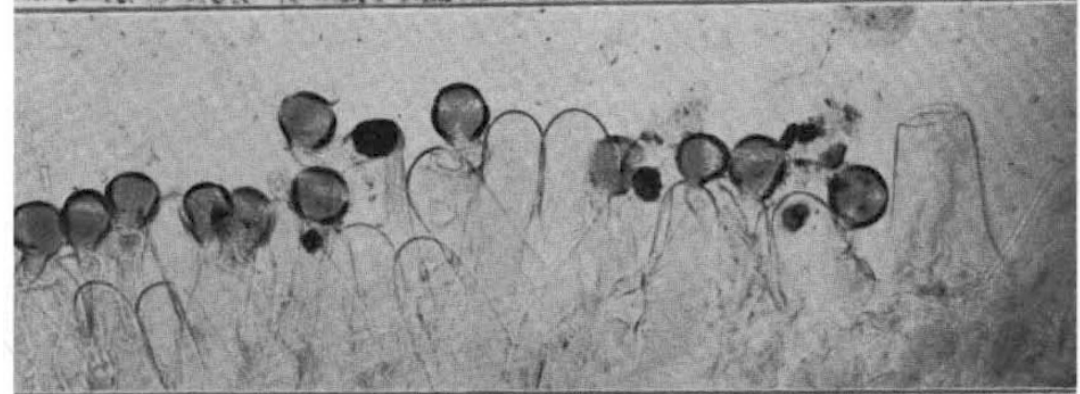

(c)

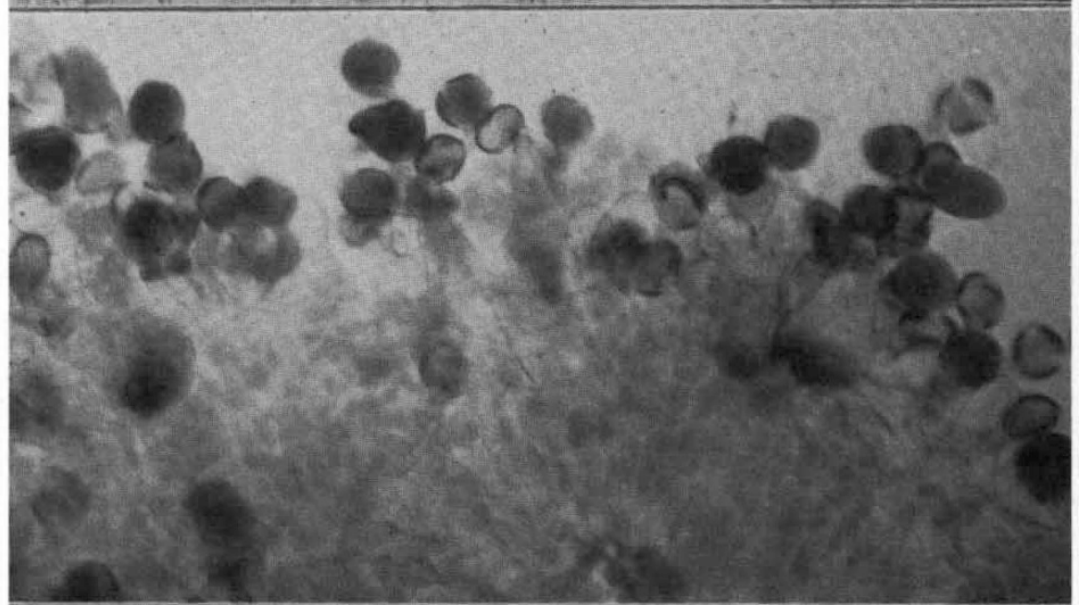

(d)

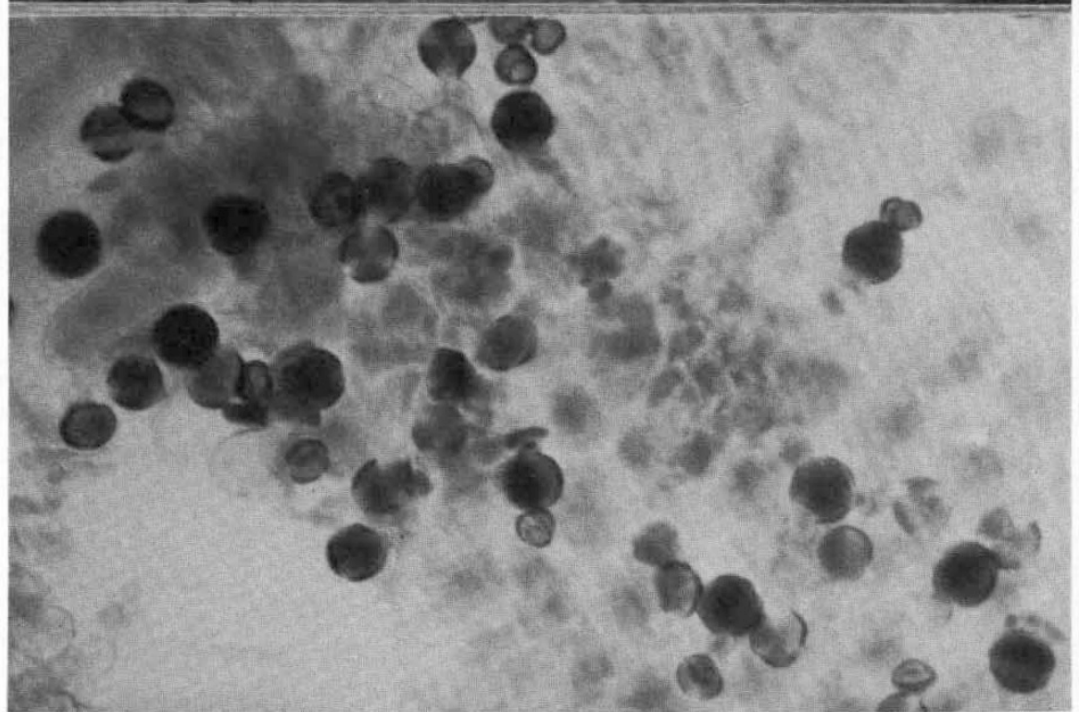

Figure 7 (a) Self-incompatible, $S$ and $G$ matched. Expected $100 \%$-pollen. (b) Cross-compatible $S$ unmatched $G$ matched or unmatched. Expected $100 \%+$ pollen. (c) Cross-compatible $S$ matched $G$ unmatched. $G_{1,1} \times G_{1,2}$, pollen lethal not present. Expected 50 per cent +50 per cent - (d) Cross-compatible $S$ matched $G$ unmatched. $G_{1,1} \times G_{1,2}$, pollen lethal present. Expected 20 per cent +80 per cent - or 80 per cent +20 per cent-dependent upon which $G$ allele is linked to $p l$. 
Table 2 Numbers of pollen grains found on stigmas after restricted pollination in four different compatible crosses

\begin{tabular}{|c|c|c|c|c|c|c|}
\hline \multirow[b]{2}{*}{ Compatible cross } & \multicolumn{2}{|c|}{ Stigma } & \multirow{2}{*}{$\begin{array}{l}\text { Washing } \\
\text { fluid }\end{array}$} & \multirow[b]{2}{*}{ Total } & \multirow{2}{*}{$\begin{array}{l}\text { Per } \\
\text { cent } \\
\text { Germ }\end{array}$} & \multirow{2}{*}{$\begin{array}{l}\text { Per } \\
\text { cent } \\
\text { E+* }\end{array}$} \\
\hline & $\mathrm{E}+$ & $\mathrm{F}$ & & & & \\
\hline $2^{2}\left(S_{3,3}\right) \times 7^{4}\left(S_{3,4}\right)$ & 382 & 83 & 23 & 488 & $95 \cdot 2$ & $82 \cdot 1$ \\
\hline $2^{2}\left(S_{3,3}\right) \times 6^{4}\left(S_{3,4}\right)$ & 463 & 39 & 127 & 629 & 73.6 & $92 \cdot 2$ \\
\hline $6^{2}\left(S_{3,3}\right) \times 6^{8}\left(S_{4,4}\right)$ & 362 & 38 & 31 & 431 & 83.9 & $90 \cdot 5$ \\
\hline $6^{2}\left(S_{3,3}\right) \times 6^{7}\left(S_{4,4}\right)$ & 199 & 125 & 169 & 493 & $65 \cdot 7$ & $61 \cdot 4$ \\
\hline Total & 1406 & 285 & & 2061 & $82 \cdot 0$ & $83 \cdot 1$ \\
\hline
\end{tabular}

* Calculated out of total on the stigma. F- full pollen grain but with penetrating tube.

\section{Self-pollination}

Twelve different plants selected from Fams 2, 3, 6 and $7 / 80$ were tested with restricted self-pollination. Since all plants are self-incompatible because the pollen and stigma have the same $S$ and $G$ alleles, there should be 100 per cent inhibited $(\mathrm{F}-$ ) pollen on the stigmas (see fig. 7(a)). Accurate counts were made on seven plants, and estimates were made where the number of pollen grains was obviously more than five hundred. The total of accurately counted pollen grains was 1432 and the estimates were all greated than 2000 . Only one doubtful $\mathrm{E}+$ pollen grain was observed; all the rest were $\mathrm{F}-$ as expected. The germination was 57 per cent, which is significantly lower than 82 per cent germination of fully compatible crosses (table 2).

\section{Cross pollinations-S matched and incompatible, $G$ active and in control}

This type of mating, where $S$ is matched and is expected on the one $S$ gene hypothesis to be incompatible, gives the exceptional +- results. We have selected $S_{3,3}$ plants from the $S$-(homogeneous) family $3 / 80$ and plants from families 6 and $7 / 80$ which segregate $S_{3,3}$ and $S_{3,4}$. From the family matings described previously we have allocated $G$ genotypes to plants as indicated in table 3. Many more restricted matings were done which are not included in the table because they were either not segregating E+ pollen or there was obvious segregation and a count was not made but recorded as +- or $1: 1$ segregation (see fig. $7(\mathrm{c})$ ). The table of results has been divided into three groups: Matings A where the male parents do not have aborted pollen, and Matings $\mathrm{B}$ and $\mathrm{C}$ where the male has 50 per cent aborted pollen. It should be noted that Group A gives approximately $1: 1$ ratio of $E+$ to $\mathrm{F}-$, Group $\mathrm{B}$ gives a large deficiency of $\mathrm{E}+$, and Group $\mathrm{C}$ gives a large excess of $\mathrm{E}+$.
Table 3 Counts of pollen grains, $\mathrm{E}+$, compatible and $\mathrm{F}-$, incompatible, after restricted pollination. Matings $A$, male parent has less than 5 per cent aborted pollen. Matings B and $\mathrm{C}$, male parent has 50 per cent aborted pollen

\begin{tabular}{lllll}
\hline & & & & \multicolumn{1}{c}{$\begin{array}{l}\text { Per } \\
\text { cent }\end{array}$} \\
Matings A & E+ & F- & E+ \\
\hline $3^{8} S_{3,3} G_{2,2} \times 7^{1} S_{3,3} G_{1,2} p L p L$ & 320 & 329 & $49 \cdot 3$ \\
$3^{8} S_{3,3} G_{2,2} \times 6^{9} S_{3,3} G_{1,2} p L p L$ & 102 & 125 & $44 \cdot 9$ \\
$2^{7} S_{3,4} G_{1,1} \times 6^{13} S_{3,4} G_{1,2} p L p L$ & 117 & 107 & $52 \cdot 2$ \\
$2^{13} S_{3,4} G_{1,1} \times 6^{7} S_{3,4} G_{1,2} p L p L$ & 142 & 156 & $47 \cdot 6$ \\
$3^{8} S_{3,3} G_{2,2} \times 7^{5} S_{3,3} G_{1,2} p L p L$ & $\sim 1: 1$ by visual inspection \\
$3^{4} S_{3,3} G_{2,2} \times 7^{2} S_{3,3} G_{1,2} p L p L$ & $\sim 1: 1$ by visual inspection \\
\hline
\end{tabular}

Matings B

$\begin{array}{llrrr}2^{7} S_{3,4} G_{1,1} \times 7^{4} S_{3,4} G_{1,2} p L p l & 96 & 215 & 30 \cdot 8 \\ 2^{7} S_{3,4} G_{1,1} \times 6^{4} S_{3,4} G_{1,2} p L p l & 62 & 272 & 18 \cdot 5 \\ 2^{7} S_{3,4} G_{1,1} \times 7^{8} S_{3,4} G_{1,2} p L p l & 26 & 48 & 35 \cdot 1 \\ 2^{11} S_{3,4} G_{1,1} \times 7^{4} S_{3,4} G_{1,2} p L p l & 25 & 107 & 18 \cdot 9 \\ 2^{11} S_{3,4} G_{1,1} \times 7^{8} S_{3,4} G_{1,2} p L p l & 13 & 78 & 14 \cdot 3 \\ 2^{11} S_{3,4} G_{1,1} \times 6^{4} S_{3,4} G_{1,2} p L p l & 13 & 70 & 15 \cdot 6 \\ 2^{13} S_{3,4} G_{1,1} \times 6^{10} S_{3,4} G_{1,2} p L p l & 28 & 118 & 28 \cdot 1 \\ 2^{13} S_{3,4} G_{1,1} \times 7^{8} S_{3,4} G_{1,2} p L p l & 24 & 73 & 24 \cdot 7 \\ 2^{13} S_{3,4} G_{1,1} \times 7^{10} S_{3,4} G_{1,2} p L p l & 19 & 122 & 13 \cdot 4 \\ 2^{13} S_{3,4} G_{1,1} \times 7^{3} S_{3,4} G_{1,2} p L p l & 11 & 72 & 13 \cdot 2 \\ 3^{4} S_{3,3} G_{1,1} \times 6^{2} S_{3,3} G_{1,2} p L p l & 550 & 1447^{*} & 27 \cdot 5\end{array}$

Mating C

$\begin{array}{lllll}3^{8} S_{3,3} G_{2,2} \times 6^{2} S_{3,3} G_{1,2} p L p l & 240 & 51 & 82.4\end{array}$

* From 5 stigmas. All crosses are $S$ incompatible and semicompatible for $G$, i.e., $G$ homozygous $\times G_{1,2}$

\section{Linkage between pollen lethal, $G$ and $S$}

The pollen counts given in table 3 can be explained on the assumption that $G$ is linked to the pollen lethal, $p l$. We have extracted, from this table, results involving plant $6^{2} / 80$ used as male in order to estimate the recombination percentage, and to demonstrate clearly the action of $G$. The figures summarised in table 4 are corrected for differences in germination of the two classes $\mathrm{E}+$ and $\mathrm{F}-$. The data are consistent with linkage between $G$ and $p l$ and a recombination frequency of about 20 per cent. It is worth noting that the plants of family $6 / 80$ and $7 / 80$ used as male in table 3 which contained $p l$ are all with $p l$ linked to $G_{2}$. If we consider the genotypes of the parents of $6 / 80$ and $7 / 80$ which are both $S_{3,4} G_{2,2} p L, p l \times S_{3,4} G_{1,2} p l$, $p L$, we see that this must be so; the only pollen to function is $G_{1} p L$. Therefore the $P l$ allele must have come from the female and, because the female is homozygous $G_{2,2}$ must be linked to $G_{2}$ allele in all progeny. The pedigree and genotypes of the plants displaying linkage of $G$ and $p l$ are given in table 5 . 
Table 4 Crosses with $6^{2}$ as male extracted from table 3 to show linkage between $G$ and $p l$

\begin{tabular}{lllll}
\hline & $\begin{array}{l}\text { Functional } \\
\text { pollen } \\
\text { grain }\end{array}$ & \begin{tabular}{lllll} 
Corrected* & Per \\
\cline { 5 - 6 } cent \\
recombination
\end{tabular} \\
\hline $3^{8} G_{2,2} \times 6^{2} \frac{G_{2} p l}{G_{1} p L}$ & $G_{1} p L$ & 296 & 89 & $23 \cdot 1$ \\
$3^{4} G_{1,1} \times 6^{2} \frac{G_{2} p l}{G_{1} p L}$ & $G_{2} p L$ & 679 & 2608 & $20 \cdot 6$ \\
$* G_{1,1} \times 6^{7} \frac{G_{2} p l}{G_{1} p L}$ & $G_{2} p L$ & 314 & 1175 & $21 \cdot 1$ \\
$3^{7} G_{1,2} \times 6^{2} \frac{G_{2} p l}{G_{1} p L}$ & none & 0 & 512 & - \\
$3^{6} G_{1,2} \times 6^{2} \frac{G_{2} p l}{G_{1} p L}$ & none & 0 & 941 & -
\end{tabular}

* The result of ten other comparable crosses, not involving $6^{2}$, are included for comparison. The correction factors, based upon percentage germination, are $\mathrm{E}+,(\mathrm{N} \times 100) / 81$ and $\mathrm{F}-$, $(\mathrm{N} \times 100) / 57$.
Linkage between $S$ and $p l$ can be estimated from fam. 6/79. It was noted by Zuberi et al. (1981) that there was a significant deficiency in the $S_{4,4}$ class (see fig. 1), and it was suggested that this could be due to a linked lethal gene. We now have evidence that this supposed lethal gene is the pollen lethal, $p l$, gene and we can use this family to estimate the recombination frequency. It is important to note that this family is the result of bud-selfing and therefore there is no restriction on the functioning of any combination of $S$ and $G$ alleles in the pollen except those carrying the gametic lethal, $p l$. A direct estimate of recombination between $S$ and $p l$ can be obtained from the twelve $S_{3,3}$ and one $S_{4,4}$ plants obtained. This gives a recombination frequency of $7 \cdot 7$ per cent.

It has not been possible to get a direct estimate of recombination between $G$ and $S$ but a useful comparison between $G-S$ and $S-p l$ recombinations can be obtained from Fams $6 / 80$ and $7 / 80$. The male parent in these families has the genotype

Table 5 Pedigree and genotypes of plants which demonstrate $G-p l$ linkage. Only $G_{2} p L$ pollen functions in the pollination that produced family $6 / 80$; this defines unambiguously the $G$ pl genotypes of the $6 / 80$ plants. The recombination frequency is obtained from the percentage of E+ pollen of $G_{1,2}$ plants of family $6 / 80$ on the stigmas of plants 4 and 8 of family $3 / 80$

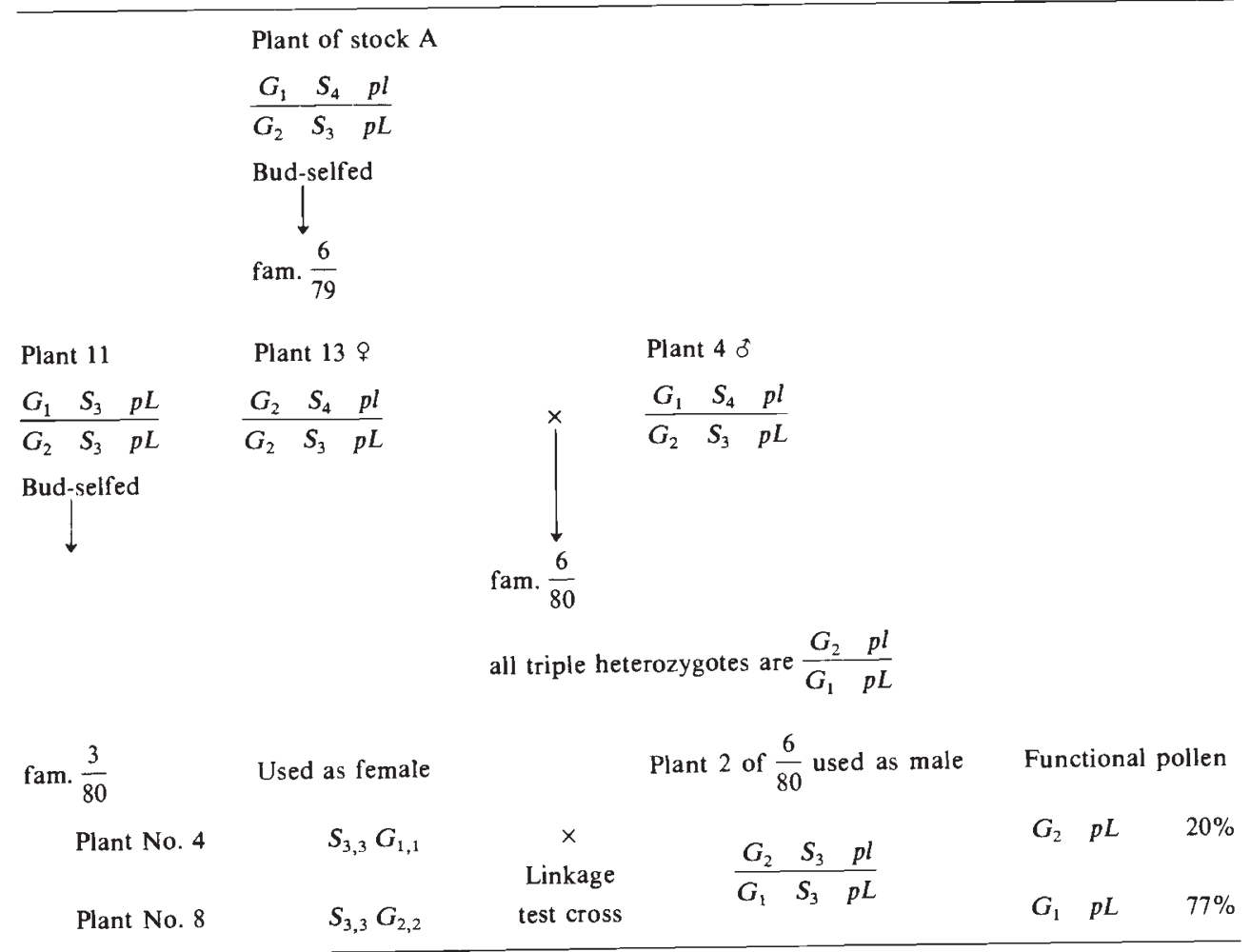


$S_{3,4} G_{1,2}, p l, p L$ and because the female parent is $S_{3,4} G_{2,2}$ the only functional pollen must have $G_{1}$ and $p L$. The two families contain a total of thirteen $S_{3,4}$, nine $S_{3,3}$ and three $S_{4,4}$. Thus from the two homozygous classes we can deduce that of the $G_{1}$ $p L$ functional pollen nine were carrying $S_{3}$ and three $S_{4}$. The recombination frequency $G-S$ is three times higher than $S-p l$. We clearly know the three genes are linked, and if we consider the three possible orders of the genes on the chromosome, there is only one which gives the two recombinants $G_{1} p L S_{3}$ and $G_{1} p L S_{4}$ which were obtained, and that is $G S \mathrm{pl}$. A rough chromosome map of the male parent can be represented as

$$
\begin{gathered}
20 \\
\frac{G_{1} S_{4} p l}{G_{2} S_{3} p L} \\
3: 1 \\
\text { say } 155
\end{gathered}
$$

If our rough estimate of $7 \cdot 7$ per cent between $S$ and $p l$ was actually 4-5 per cent we arrive at a consistent estimate of recombination frequencies and an unequivocal order of the genes.

\section{DISCUSSION}

\section{Evidence for gametophytic action of the G gene}

The strength of the evidence for the gametophytic action of the $G$ gene in Brassica campestris rests on the genetic diversity of the nine families studied, rather than on a complete mating pattern within a family which excluded a sporophytic gene in Raphanus sativus (Lewis et al., 1988). This difference is partly due to fortuitous choice of parent plants and partly due to the larger families studied in Raphanus. The summary of the results from the nine families is given in table 6. There are four families in which there are no +- exceptions caused by $G$ compatibility. One of these families is due to selfing a $G$ homozygote, one to crossing two different homozygotes to give all progeny with $G_{1,2}$ and two families are crosses where the male parent is $G_{1,2}$. In these test families $G$ is in control in the pollen making one of the two $G$ alleles incompatible so that the other allele is the only one passed to the next generation. All the remaining five families have +- anomalies as expected, because $G_{1,2}$ and $G$ homozygotes should be present in the family.

The most convincing evidence comes from a comparison of fam. $4 / 80 G_{2,2} \times G_{1,2}$ and $S$ un-
Table 6 Summary of +- results due to $G$ compatibility. The main significance is whether +- results are or are not obtained; all ten families agree fully with expectations. $G_{1}$ functional on $G_{2,2}$ style

\begin{tabular}{lll}
\hline & Fam. & $\begin{array}{l}+- \\
(G \text { compatibility })\end{array}$ \\
\hline $\begin{array}{ll}\text { Bud pollinations } \\
S \text { and } G \text { neutralized }\end{array}$ & $\begin{array}{l}1 / 80 G_{2,2} \text { selfed } \\
2 / 80 G_{1,2} \text { selfed }\end{array}$ & 0 \\
& $3 / 80 G_{1,2}$ selfed & 2 \\
& & \\
Cross pollination & & \\
$S$ compatible, $G$ silent & $4 / 80 G_{2,2} \times G_{1,2}$ & 7 \\
& $5 / 80 G_{1,2} \times G_{2,2}$ & 6 \\
& $8 / 80 G^{2} \times G_{2,2}$ & 4 \\
& $9 / 80 G_{1,1} \times G_{2,2}$ & 0 \\
Cross pollination & & \\
$S$ matched $G$ active & $6 / 80 G_{2,2} \times G_{1,2}$ & 0 \\
& $7 / 80 G_{2,2} \times G_{1,2}$ & 0 \\
\hline
\end{tabular}

matched (compatible), so that both $G_{1}$ and $G_{2}$ are transmitted, and the fams $6 / 80$ and $7 / 80 G_{2,2} \times$ $G_{1,2}, S$ matched (incompatible) where only $G_{1}$ will be transmitted. The presence of +- matings in fam. $4 / 80$ and their absence in $6 / 80$ and $7 / 80$ completely confirm this consequence of the gametophytic action of $G$ and also its role as a complementary oppositional factor to $S$.

One interesting consequence of this action is that, if families are raised from exceptional +matings, they will not show these +- types of matings in the next generation.

The +- and - pollen counts, together with the linkage of $G$ to a pollen lethal $p l$ and to $S$, are a different but equally compelling evidence for the gametophytic action of $G$.

The similar linkage of $G$ with $S$, (best estimate in Brassica 15 per cent recombination, in Raphanus 25 per cent) emphasis that the $S-G$ system has long been established, for Brassica campestris and Raphanus sativus are not close - Brassica with $2 n=20$ and Raphanus $2 n=18$. The genes have remained together despite the chromosome differences.

\section{G expression}

The $G$ gene appears to be present in all the plants of Brassica and Raphanus that we have examined, and we presume this must be so by its complementary action to $S$, but it does not express its presence in all $S$ genotypes. Within the combinations of $S_{3}$ and $S_{4}$ obtained from the one bud-selfed family $6 / 79, G$ is expressed fully or sporadically in all but one, which was not adequately tested, of the 
crosses. These include homogenic crosses, e.g., $S_{3,4} \times S_{3,4}$. With Raphanus, Lewis et al. (198) $G$ expression was confined to certain heterogenic crosses, e.g., $S_{1,2} \times S_{1,4}$. However, limited $G$ expression is found in Brassica by crossing into a different $S$ genotype. A family of twenty-three plants raised from a cross between the parent of fam. $6 / 79$ to a plant of the same stock but with $S_{1}$ and $S_{2}$ produces no +- exceptions. The number of incompatible matings tested was 672 and without a single exception all the plants had either $S_{1}$ or $S_{2}$ and $S_{3}$ or $S_{4}$. Both the $G$ alleles derived from the $6 / 79$ parent would be transmitted, and whatever the $G$ constitution of the other parent, there should be $G_{1,2}$ and homozygotes segregating. We presume that the recognition distinction between $G_{1}$ and $G_{2}$ is suppressed in the presence of $S_{1}$ and $S_{2}$.

The conditional expression of $G$ in Brassica agrees in general with the comparable results in Raphanus and strengthens the view that $G$ with its limitation to two alleles is a relic of an old system which is now retained for its essential part in the pollen-stigma recognition system. It has, however, lost full expression of its own alleles which is part of the system that is no longer required to generate multiple mating groups. These are adequately supplied by the multiple alleles of $S$.

Acknowledgements The authors express their thanks to the Leverhulme Trust, the Royal Commission for the Exhibition of 1851 and the Royal Society for support grants.

\section{REFERENCES}

CORNISH, M. A., HAYWARD, M. D. AND LAWRENCE, M. J. 1979. Self-incompatibility in rye grass. I. Genetic control in Diploid Lolium perenne L. Heredity, 43, 95-106.

LEWIS, D., VERMA, S. C. AND ZUBERI, M. I. 1988. Gametophytic-sporophytic incompatibility in the Cruciferae Raphanus sativus. Heredity, 61, 355-366.

SAMPSON, D. R. 1962. Intergenic pollen-stigma incompatibility in the Cruciferae. Can. J. Genet. Cytol., 4, 38-49.

TATEBE, T. 1940. Studies on the behaviour of incompatible pollen in Japanese radish. J. of Hort. Ass. Jap. 11, 207-234.

ZUBERI, M. I., ZUBERI, S. AND LEWIS, D. 1981. The genetics of incompatibility in Brassica. I. Inheritance of self-incompatibility in Brassica campestris L. var. Toria. Heredity, 46, $175-190$. 